\title{
Organizational climate aspects and principal's burnout in Southern Israel schools
}

\author{
Andreea Ionică ${ }^{1, *}$, Yunnis Nassar $^{2}$, and Sorin Mangu ${ }^{1}$ \\ ${ }_{1}$ Management and Industrial Engineering Department, University of Petrosani, Romania \\ ${ }^{2}$ Management and Industrial Engineering Department, Doctoral School, University of Petrosani, \\ Romania
}

\begin{abstract}
The scientific literature abounds in researches about the impact of the organizational climate on burnout among teachers, but the topic wasn't explored enough at the principals' level till the results of the researches of Friedman and Kremer-Hayon were published. These are considered the main premises of the research in this paper which reports on a quantitative research study. The proposed toolset consists of questionnaires validated in the literature, demonstrating stable reliability coefficients and also, being validated in the educational contexts and systems from Israel. Three types of questionnaires are used: one to determine the individual perception of the principals' communication with the teachers, one to determine the organizational climate and another questionnaire for burnout, addressed to the school principals. In the crosssectional research, the Israeli Arab teachers who participated in this study were selected from the sample of four schools from South Israel $(22 \%$ of total schools) obtaining 120 validated questionnaires. The results of the research highlighted a moderate level of burnout. However, the biggest values appear on the dissatisfaction dimension as long as there is a positive communication, medium to high harmony and interpersonal relationships and a medium level of trust and support among teaching staff.
\end{abstract}

\section{Introduction}

The existence of occupational psycho-socio risks poses permanent challenges in the area of occupational health and safety due to the significant incidence of workplace problems such as work-related stress and burnout. Although there is sufficient information in the literature on the adverse effects of burnout on employees, the information on organizational strategies that can play a role in reducing or preventing them is very low. This is why the existing research focuses mostly on the relationship between the organizational climate and the burnout syndrome among employees. The education sector is considered to be among the areas exposed to burnout. A series of researches focused on teacher's burnout issues, but a less explored area is that of the school principals. This research has a high degree of specificity because it pertains to the perceptions of the communication between the

*Corresponding author: andreeaionica@upet.ro 
principal and the teachers in terms of positive, negative and lack of communication sequence, explores the dimensions of the organizational climate (the level of harmony in the educational staff, interpersonal relationships, the level of trust and support in staffing), the relationship between perceptions of interpersonal relationships (as a dimension of the school climate) and the burnout level of the principals of the Arab schools in Southern Israel. So, the defined field of research is impregnated by highly individualized characteristics of the Arab education system in Israel.

\section{Literature review}

The last decades have been marked by major economic, political, social and cultural changes, with a profound impact on organizations' activities and implicitly on the human resources involved, sometimes manifested as depression, panic disorder, mental disorders and stress disorders strongly related to the professional working environment. According to the well-known definition provided by Maslach C., burnout is a psychological syndrome described as a specific response to prolonged exposure to stressors at work, and has three components: exhaustion, depersonalization, and reduced self-efficacy.

There is a relationship with the psycho-social factors of the job-specific conditions, namely the work tasks organization, the content of the type of tasks and the expected performance of the employees [1]. In this sense, burnout can occur when there is an imbalance between the job requirements and the availability of the employee's personal resources [2], when great efforts are involved to perform the tasks [3], when conflicts of interest take place between employees, and also between organizational missions and career guidance [4] appear. Burnout syndrome has become a subject of increasing interest in psychology and psychiatry, being first described in the mid-1970s by Freudenberger, and later by Maslach and Pines. In general, viewed as a work-induced disorder [5] this concept has also become a topic of interest in occupational safety and health. The syndrome has been associated with a variety of negative professional consequences - including decreased work performance and absenteeism [6,7]. Research on burnout has been intensified in recent years, thus various recommendations and demands on the management of occupational stress have emerged. The term burnout, without the necessity of a specific definition, attempts to integrate the symptoms (fatigue, emotional exhaustion, reduced personal achievement and distance from colleagues), as well as causes related to the level of tasks' achievement. Practical and theoretical research has provided a number of approaches that have been used to describe, explain and predict burnout syndrome [8-11]. The researches have shown that factors that influence the occurrence of burnout at the workplace are both at the individual and organizational level. Thus, the dimensions of the organizational climate becomes relevant in the context of studying the occurrence of burnout syndrome. The literature provides an inventory of methods and tools used to assess the relationship between the organizational climate and the burnout syndrome based on researches in areas considered to be the most exposed (education, health and social care).

The approaches to burnout syndrome in education focused on issues such as the relationship between: emotional intelligence and burnout [12], work-related stress and burnout syndrome in schools [13-20]

\section{Context of the research}

The specificity of the Israeli educational system leads to distinctive theoretical and practical approaches. So, among the particular aspects that determine the occurrence of burnout, the following predictors can be included: the impact of the educational reforms, the 
delimitation of two systems, namely the Jewish Israeli educational system and the Arabic Israeli educational system, the content of didactic materials [21], the existence of a significant violence rate, the impact of terrorism [22].

Cultural complexity adds significant aspects to contextualizing the research regarding the factors that contribute to the performance of teachers in a highly specific organizational climate. The schools in Israel form a traditional Middle Eastern community based on a Western organizational model impregnated by the traditional aspect of the community. The existence of this mixed culture according to the factors that influence the organizational climate and how the satisfaction of the teachers is affected is understood and interpreted in the organizational research from the perspective of interculturalism.

Literature has shown that the understanding of the relationship between organizational climate and work satisfaction has become vital to facilitating organizational development. Identifying the dimensions of these variables and measuring them is a means of determining the status of an organization and can help improve the school climate. However, a limited number of studies on the organizational climate have been made in modern Arab societies and schools in the Arab education system, respectively. In general, research demonstrates a vast and often conflictual range of influences on organizational development. There are a number of historical factors that influence modern Arabian organizational practices, including Islamic traditions and values, tribal and family traditions, the legacy of colonial bureaucracy, increased contact with the West in recent decades, government intervention, and political constraints.

Recent studies [23] examine the relationship between teacher motivation as a dependent variable and management style and educational climate as independent variables. Previous research supports the predominant role of the principal in Arab schools in Israel. Most research on the occurrence of burnout was focused on teachers and the phenomenon of burnout of the school principals was not a topic explored sufficiently until Friedman [24] and Kremer-Hayon, Faraj \& Wubbels [25] researches that constitute the premises of the research in this paper. The results indicated a relatively low level of burnout faced by Israeli Arab school principals both generally speaking and compared to school principals from the Jewish education sector. There was established a principal profile determined by a certain social status characterized by parents and community respect. The premises of the research are: changes in the principals' profile that can lead to burnout, along with the existence of gaps between principals' and teachers' perceptions about the interpersonal relationships (existing and desirable), due to cognitive dissonance.

\section{Methods and materials}

The methodological toolset includes questionnaires demonstrating stable reliability coefficients and also, being validated in the educational contexts and systems from Israel. There are used questionnaires in order to determine: the individual perception of the principal's communication with the teachers, dimensions of the organizational climate [26] and the level of burnout of the school principals [27].

For individual perception of the principal's communication with the teachers, the questionnaire is used with the purpose to examine the communication perceptions between the principal and the teacher in positive, negative and lack of communication sequence. The questionnaire's items were divided into three groups: positive communication, negative communication and lack of communication. The answers' categories are in order scale between - 1 - very correct and 5 - absolutely wrong. The Cronbach's alpha reliability of the three types of communication are: positive $\alpha=0.93$, passive/lack $\alpha=0.92$, negative $\alpha=0.93$. The data were recoded to perform a comparative analysis of the three components of the 
study. Thus, the variables scale was from 1 to 5 , in which 1 notes the lowest value and 5 notes the highest value.

School climate questionnaire was built based on chosen items of different questionnaires dealing with school climate [26]. The questionnaire has three dimensions: the level of harmony in the educational staff, interpersonal relations, the level of trust and support among the staff. The participants were asked to answer in a 5 degrees Likert scale, when 1 represents the level in which the statement in its lowest level and 5 represent the highest level. The general Cronbach's alpha reliability for the whole scale was $\alpha=0.70$, and for the sub-scales as follows: the level of harmony among the educational staff $\alpha=0.87$, interpersonal relations $\alpha=0.62$, the level of trust and support among the staff $\alpha=0.84$.

This questionnaire for burnout, addressed to the school principals Friedman (1996), consisted of 22 items containing the dimensions: exhaustion, isolation, dissatisfaction with oneself, and dissatisfaction with colleagues. The scale's reliability, as expressed in the items' homogeneity and the internal traceability, was found through Cronbach's alpha coefficient procedure. The alpha coefficient of the whole scale is relatively high for 22 items $(0.92)$. The sub-scales alpha coefficients are: exhaustion 0.90 , segregation 0.84 , and dissatisfaction with others 0.77 .

The Israeli Arab teachers who participated in this study were selected from the sample of 4 schools from South Israel, (representing $22 \%$ of total schools) that mean a total of 142 teachers aged between 22 and 62, obtaining a number of 120 validated questionnaires.

In the first phase of the research, the application, the analysis and the interpretation of the questionnaires were made at the level of each school. There were no significant differences between the results achieved at each school level, which allowed in the second phase of the research that the processing and the interpretation of the results to provide an overview of the issues concerning the communication between teachers and the school principal, the dimensions of the organizational climate (harmony, relationships interpersonal and trustworthy), respectively the level of burnout of principals. A crosssectional research has been done using a stratified sampling.

\section{Results and discussions}

\subsection{Principal's communication with the teachers}

The perceptions concerning the principal's communication with the teachers, were analysed in terms of positive (Figure 1), passive/lack of communication (Figure 2) and negative communication sequence (Figure 3).

The average value of the positive communication between the principal and the teachers is between "medium level" and "high level" $(\mathrm{M}=3.27)$. The average of the passive/lack communication between the principal and the teachers is between "low level and "medium level" $(\mathrm{M}=2.38)$ and the average of the negative communication between the principal and the teachers is also, between "low level" and "medium level" $(\mathrm{M}=2.67)$. Therefore, it can be assumed that in most cases the teachers tend to classify the communication between them and their principals as positive and less as negative and/or passive.

\subsection{School climate dimensions}

The organizational climate analysis was carried out on three dimensions: the level of harmony among the educational staff (Figure 4), interpersonal relations (Figure 5), and the level of trust and support among the staff (Figure 6). 
Willing to help teachers in their additional assignments bey ond teaching Positively guiding teachers in improving their work

Characterized by positive human relations

Interested in the teachers' personal lives

Take part in family events of his teachers

Shows positive attitude towards teachers who have personal family constraints

Initiate dialogs with the teachers about curriculum updates and improvements

Initiate empowering dialogs with the teachers' staff

Initiate dialog to the staff's social bonding

Expresses praise to teachers about their successful functioning

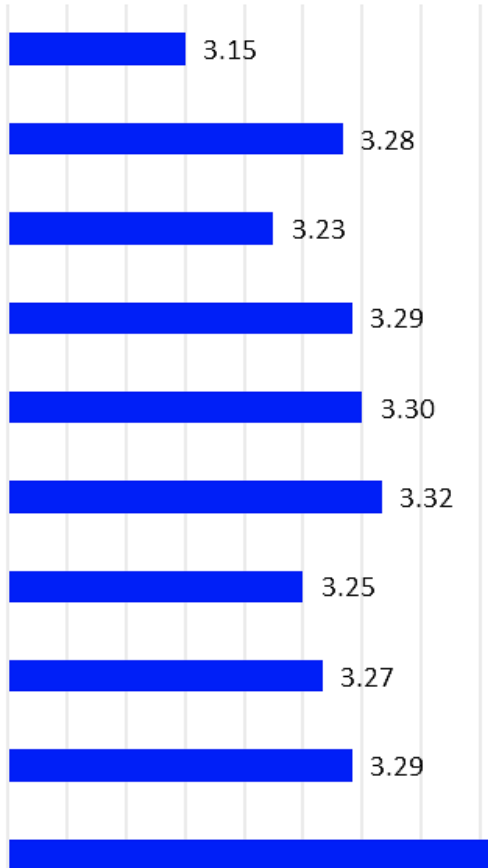

3.41

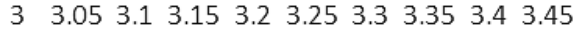

Fig. 1. Positive communication aspects in the relationship between principals and teachers.

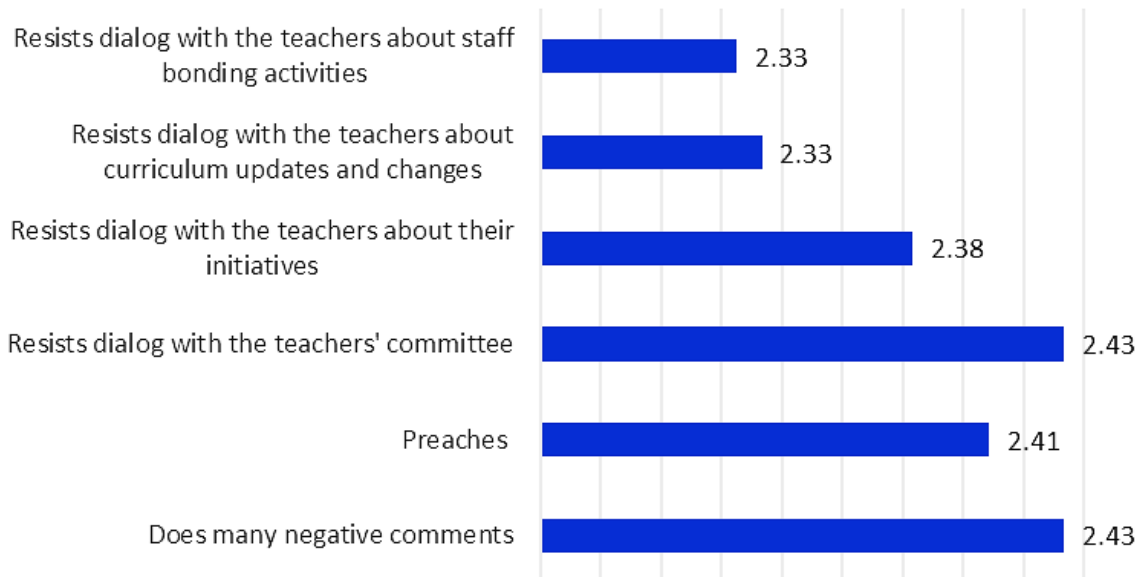

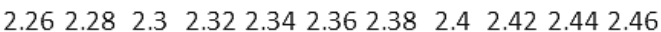

Fig. 2. Lack of communication aspects $n$ the relationship between principals and teachers. 
Expresses dissatisfaction of the students' achievements

Negatively criticize teachers about their work

Avoid responding the variety of events in school

Avoid communication with teachers in their additional assignments beyond teaching

Does not have constant relation with the teachers

Avoid evaluating teachers' work

Does not communicate with teachers about school's social activity

Does not communicate with teachers in their teaching subjects

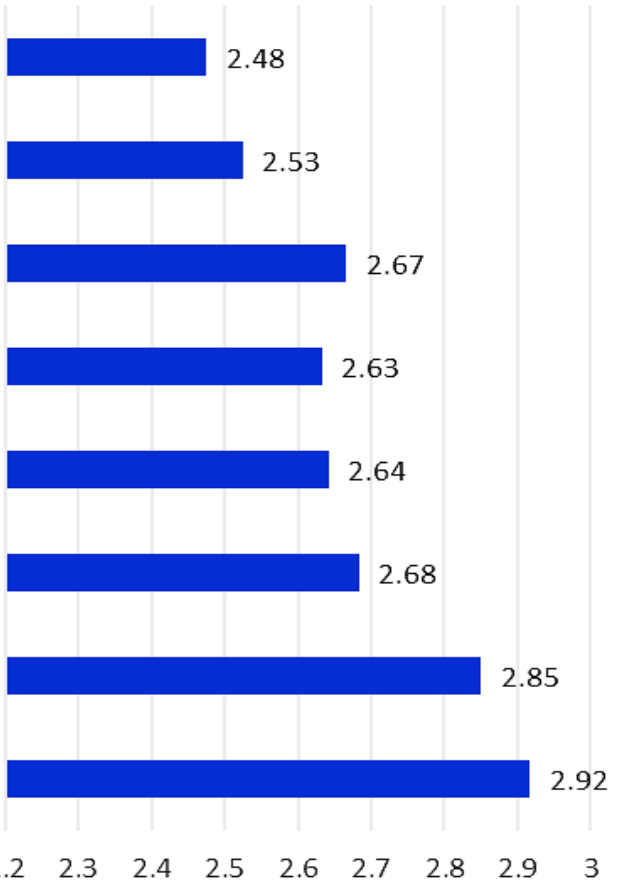

Fig. 3. Negative communication aspects in the relationship between principals and teachers.

There is mutual help among the educational staff when needed

We see great importance to professional cooperation between the staff members

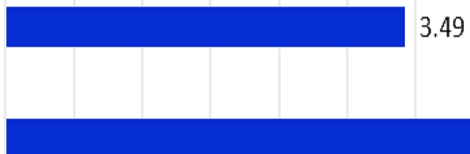

There is an atmosphere that encourage cooperation in information and ideas among the educational staff...

The personnel members in our school are organized in teams which work in cooperation

There is high loyalty among the educational staff members

The educational staff members meet also after working hours

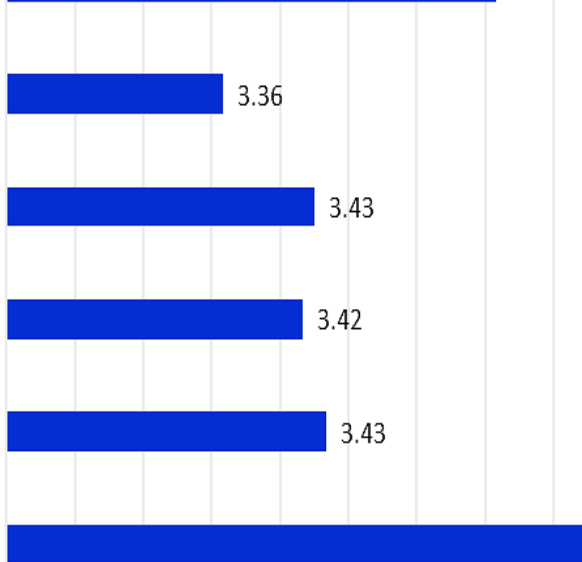

The teachers' room is a pleasant place

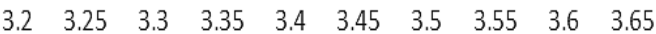

Fig. 4. The level of harmony among the educational staff. 


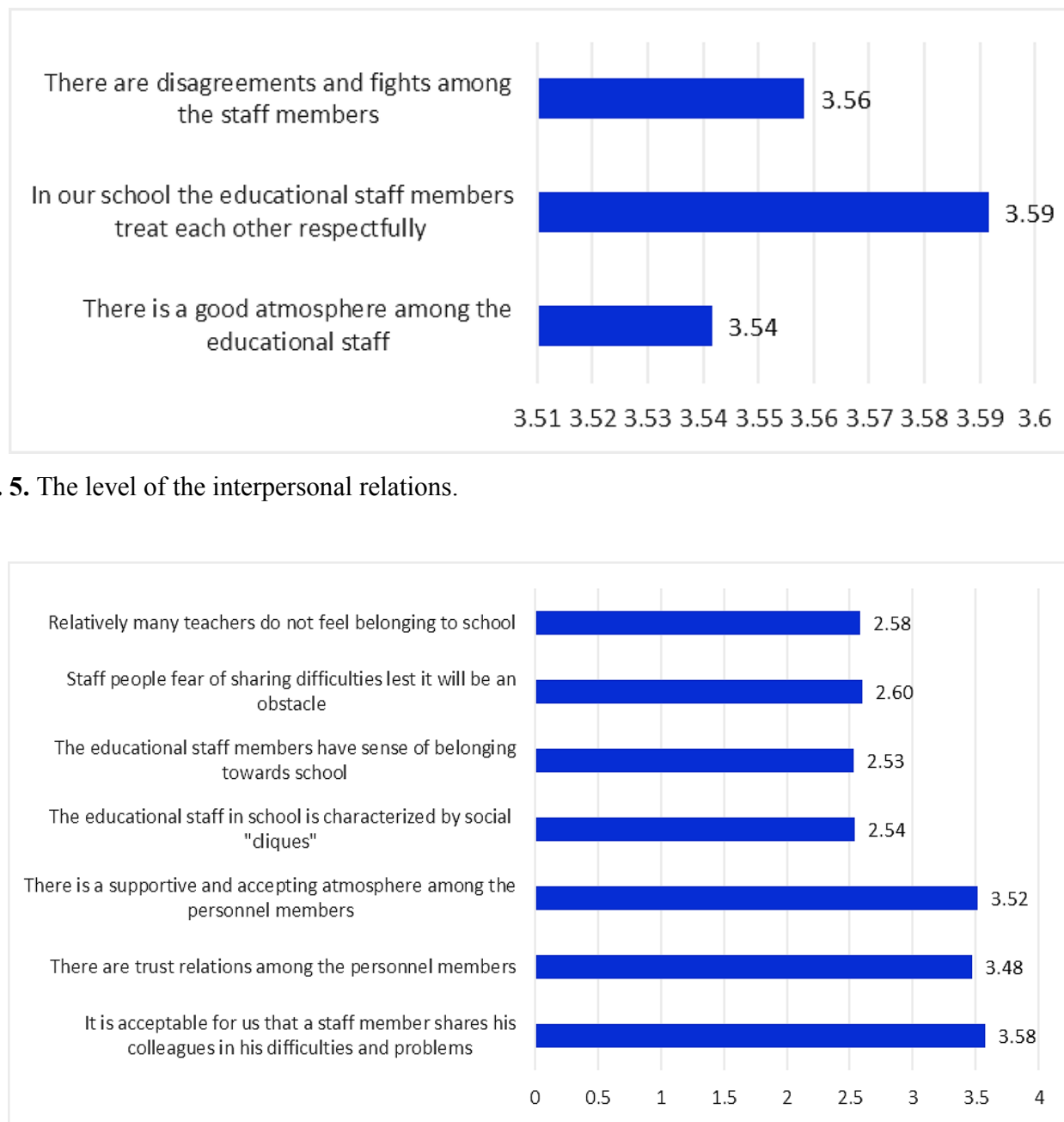

Fig. 6. The level of trust and support among the staff.

For all the dimensions of the climate analysis, were obtained values around the medium level. On the Harmony among teachers dimension and Interpersonal relations, the levels are considered from "medium" to "good" $(\mathrm{M}=3.47$ and $\mathrm{M}=3.56)$. The level of trust and support among the staff is considered about the "medium" level $(\mathrm{M}=2.97)$.

An interpretation would lead to the need to improve the three dimensions of the analysed climate and, in particular, the interpersonal relations where the item concerning the existence of disagreements and battles among staff members appears with an average that exceeds the medium level $(M=3.56)$, in the conditions of the existence of a harmony among teachers appreciated over the medium level and the trust and support among the staff expressed around the medium level. Next, will be examined whether the conditions given by the exposed climatic dimensions are appropriate for the principals' burnout state occurrence. 


\subsection{Principals' burnout level}

The school principal's burnout level analysis was carried out on the dimensions: Exhaustion, Segregation and Dissatisfaction with others (teachers, students and parents) (Figure 7).

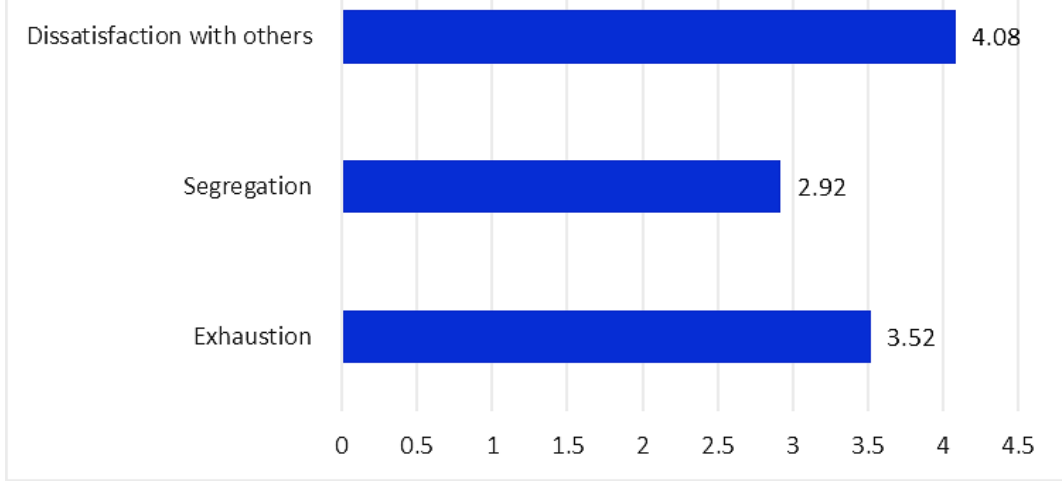

Fig. 7. The level of burnout among principals.

The nine items of the Exhaustion sub-scale describe feelings of physical, emotional and conscious tiredness describing the principal's loss of energy. In this dimension, the first three items refer to the principal's exhaustion originating in managing and operating the school. The average value for these three items $(\mathrm{M}=3.58)$ is higher than the average for the whole Exhaustion dimension ( $\mathrm{M}=3.52)$

The seven items in the Segregation sub-scale describe the principal's distance feeling from the teachers (or other persons involved) and his keeping away from problems, ideas and new suggestions. Most of the items in this sub-scale point that this distance keeping of the principal is against his feelings/sensations and his past behaviour, reducing the principal's enthusiasm of his work, his dedication to his role and his support among his employees.

The Dissatisfaction sub-scale includes six items describing negative feelings expressed in diminished the ability of the principal's service recipients, the employees and those in his environment: teachers, students, parents. These items express the principal's awareness that those who work with him do not function as they were, including the relations with the students and with their parents. The highest values were obtained concerning the following aspects (items): I think that teachers bring to me many problems they could have solve by themselves ( $\mathrm{M}=4.25)$, I think I would like that in my school were better teachers then those who work in it now $(\mathrm{M}=4.25)$, I feel I have no satisfaction of the teachers' functioning as I used to have $(\mathrm{M}=4)$, I think that students in my school does not properly invest in their studies $(\mathrm{M}=4.25)$, I think the parents complain to me about subjects or problems they are responsible for $(\mathrm{M}=4.25)$.

Higher average grade in all sub-scales and the entire principal's burnout scale refers to higher level of burnout experienced by the principal.

\section{Conclusions and further enhancements}

The research findings revealed that the state of principal's burnout may be moderated, while the main and central mission is to improve the principal's managing skills concerning the maintaining of a positive climate (positive communication, harmony and trust among the staff), and getting better cooperation with the teachers, students and parents. For the 
Exhaustion dimension of burnout, there comes to the forefront the results referring to the principal's exhaustion originating in managing and operating the school with a higher value $(M=3.58)$ than the average for the whole Exhaustion dimension $(M=3.52)$. The moderate values $(\mathrm{M}=2.92)$ of the Segregation dimension of the burnout state has to be corroborated for further interpretation with the positive communication results obtained over the medium (M 3.27), attention is drawn by the results for the item concerning the fact that the principal feels that the relations with the teachers and students are less personal then they used to be, for which there have been obtained high average value $(\mathrm{M}=4.00)$, pointing out a potential identified source of principals' burnout in the future, the nature of relations with the teachers and students. The highest values for the principals' burnout state appears on the dissatisfaction component $(\mathrm{M}=4.08)$, in the context of a positive communication $(\mathrm{M}=3.27)$, harmony among teachers' staff $(\mathrm{M}=3.47)$ and interpersonal relations $(\mathrm{M}=3.54)$ appreciated around the medium level. This means that the pressure on the principals comes from teachers, students and parents. Teachers do not feel a negative communication, lack of harmony or lack of support from managers, but their problems have an impact on principals, given the fact that were identified "signs" of disagreements and even fights among staff members, along with student and parent-related components.

The results obtained in this study highlight aspects that constitute the premises of further research. These will aim to increase the research area, which was initially established at four schools from districts from Southern Israel (Ber Mshash, Abutal, Elbaian, Aatam). Investigating a larger population (both for managers and teachers) can lead to more accurate and relevant findings in order to express the trends that might be useful for estimating burnout levels for school principals. Given the high degree of specificity of the area, in the next stage of the research a profile of the school principal will be established based on the influence of stressors (environmental or induced) that can lead to significant levels of burnout. In further researches a designed wearable device (prototype stage) will be used for biometrical data acquisition and early burnout trend prediction. The present state of our research in this area is the biometric identification [28,29] and the conceptual design of burnout evaluation system based on the identified biometric signals [30]. The device will be calibrated using both the principal's responses at the questionnaires and the physiological parameter values.

\section{References}

1. P. R. Gil-Monte, Eur Psychol J.17(3), 231-236 (2012)

2. A. B. Bakker, E. Demerouti, J Occup Organ Psychol., 29, 107-115 (2013)

3. W. B. Schaufeli, IRSP, 19, 87-131 (2006)

4. M. P. Leiter, A. B. Bakker, C. Maslach, Burnout at work, (Taylor \& Francis, 2014)

5. W. B. Schaufeli, T. W. Taris, Work Stress, 19 (3), 256-262 (2005)

6. B. W Swider, R. D. Zimmerman, J Vocat Behav, 76(3), 487-506 (2010)

7. S.Toker, S. Melamed, S Berliner, D. Zeltser, I. Shapira, Psychosom Med. 74(8), 840-847,(2012)

8. R. Karasek, B. Choi, P.O. Ostergren, M. Ferrario, P. De Smet, P. Int J Behav Med, 14(4),189201 (2007)

9. J. Siegrist, J Occup Health Psychol, 1(1), 27-41, (1996)

10. C. Maslach, W.Schaufeli, M. Leiter, Annu Rev Psychol, 52, 397-422 (2001)

11. D.Camerino, M. Cassitto, M. Gugiari, P. Conway, P. Med Lav, 104, 411-427 (2013)

12. M. Mohammadyfar, J.of the Indian Academy of Appl. Psycho, 35 (2), 219-226 (2009)

13. J. M Lopez, IJP\&PT, 10(1),107-123 (2010)

14. R. Yusoff, Res. j. recent sci., 2(11), 90-98 (2013)

15. S. M. Johnson, IJTARP, 6 (1), 70-85 (2015)

16. G. Reddy, IJEPA, 3(1), 9-24 (2013) 
17. G. C. Mahakud, IJEPR 3(2) (2014)

18. Russell, J., Stress Free Teaching, A Practical Guide to Tackling Stress in Teaching, Lecturing and Tutoring. (EDP Kogan Page Limited, 2000)

19. N. Tsigilis, E. Zachopoulou, V. Grammatikopoulos, Educ. Res. Rev. 1(8), 256-261(2006)

20. M. Brackett, R. Palomera, J. Mojsa-Kaja, M.R. Reyes, Psychol Sch, 47(4) (2010)

21. C. Hamilton, Family, Law and Religion. (Sweet \& Maxwell 1995)

22. A. Thapa, J. Cohen, S. Guffey, A. Higgins-D’Alessandro, Rev Educ Res, 83(3), 357-385 (2013).

23. Z. Accariya, M. Khalil, CE, 7, 1995-2010 (2016)

24. I. A. Friedman, Soc Psycho of Edu, 5, 229-251 (2002)

25. L. Kremer-Hayon, H. Faraj, T., Wubbels, Int. J of Leadership in Ed, 5 (2), 149-162 (2002)

26. R. Maslowski, JEA, 44 (1), 6-35, (2006)

27. I. A. Friedman, Stress and Coping, 9, 245-259 (1996)

28. A.C. Ionica, M. Leba, Procedia Economics and Finance, 23, 986-991 (2015)

29. O. M. Musetoiu, M.Leba, A. C. Ionica, I. J. of Applied Mathematics and Informatics 12, 51 (2018)

30. Y. Nassar, R. Marcus, A. C. Ionica, M. Leba, IJES, 6, 95 (2018) 